\title{
Zum 90-jährigen Bestehen der VHN
}

\author{
Birgit Herz \\ Leibniz Universität Hannover
}

Gegründet 1932 mit dem Titel Heilpädagogische Werkblätter, feiert die VHN - Vierteljahresschrift für Heilpädagogik und ihre Nachbargebiete ihr 90-jähriges Bestehen und ist damit die älteste wissenschaftliche Fachzeitschrift für Heil- und Sonderpädagogik im deutschsprachigen Europa.

Bereits seit 1908 war die Zeitschrift Die Hilfsschule des Verbandes der Hilfsschulen Deutschland mit einem monatlichen Umfang von zunächst eineinhalb Bogen bei der Carl Marhold Verlagsbuchhandlung in Halle a.d.S. erschienen. Dieser "Newsletter" des Verbandes der Hilfsschulen Deutschland wurde von 1934 bis 1945 unter dem Titel Die deutsche Sonderschule als Organ der Reichsfachschaft Sonderschulen im Nationalsozialistischen Lehrerbund (NSLB) publiziert; er war freilich - wie der Titel sagt - schulpädagogisch orientiert. Ab 1949 erfolgte mit dem Titel Heilpädagogische Blätter eine Neugründung; ab 1951 erschien im Auftrag des Verbandes Deutscher Hilfsschulen die Zeitschrift für Heilpädagogik (vgl. Herz, 2017).

Dieser kurze Verweis auf an vergleichbare Zielgruppen adressierte Publikationsorgane soll deutlich machen, wie groß zu Beginn des letzten Jahrhunderts das Interesse und die Nachfrage nach Informationen, Praxiserfahrungen und wissenschaftlichen Erkenntnissen vonseiten der Fachöffentlichkeit waren. Der Blick in die Geschichte zeigt allerdings auch, dass die damaligen Verbindungen zu faschistischem Gedankengut noch kaum systematisch erforscht wurden.
Dies gilt wohl ebenso in Bezug auf Prof. Josef Spieler, unter dessen Regie das erste Heft der Heilpädagogischen Werkblätter 1932 am Institut für Heilpädagogik in Luzern erschien, als auch für die Gründungsväter des Verbandes der Hilfsschulen Deutschland - und damit des Verbandsorgans: Eine Aufarbeitung ihrer Positionierungen und Profilierungen im Nationalsozialismus fehlt, zumindest im ganzheitlichsystematischen Sinne.

Urs Haeberlin konnte nachweisen, dass Spieler bereits während seiner Schweizer Zeit als Hochschullehrer in Luzern und Fribourg Mitglied der NSDAP war und trotzdem noch 1976 den Verdienstorden des Landes Baden-Württemberg erhielt. Spieler hatte nach seiner Ausweisung aus der Schweiz im Jahr 1945 als Professor und Rektor der Pädagogischen Hochschule Karlsruhe gewirkt (vgl. Haeberlin, 2016, S. 279f.).

Nach dem Zweiten Weltkrieg übernahm Prof. Dr. Eduard Montalta als neuer Institutsdirektor des Heilpädagogischen Instituts der Universität Freiburg/Fribourg die Herausgabe der Heilpädagogischen Werkblätter. Mit dieser personellen und institutionellen Etablierung zeichnete sich bereits vor über einem halben Jahrhundert ab, dass die Anfänge und historischen Kontinuitäten sehr eng verknüpft sind mit der Entwicklung der Sonder- und Heilpädagogik in Fribourg. Am Institut lokalisiert war nämlich auch das gesamte Management dieser bereits in den 1950er Jahren renommierten Fachzeitschrift, die sich 1971 einen neuen Namen gab: Aus den Heilpädagogischen Werkblättern wurde die VHN, die Vierteljahresschrift für Heilpädagogik und ihre Nachbargebiete. 
Als Urs Haeberlin 1979 in der Hochphase pädagogischer Reformbestrebungen die Nachfolge von Eduard Montalta auf dem Lehrstuhl für Heilpädagogik antrat, zeichnete sich bereits ab, dass er auch die Herausgabe der VHN übernehmen würde.

Haeberlin war als Wissenschaftler eng vernetzt mit Kolleg/innen aus der Bundesrepublik Deutschland und Österreich, insbesondere auch im Kontext der in den 1980er Jahren in Frankfurt am Main gegründeten Integrationsforschergruppe. Er übernahm die VHN in einer Epoche, in der polarisierende Kräfte-Krüppelbewegung, Gentechnologie, Pränataldiagnostik, kategoriale versus nonkategoriale Sonder(schul)pädagogik u. Ä.m. - das Fach herausforderten.

Ein integrationspädagogischer Diskurs - bei gleichzeitiger Etablierung neoliberaler Steuerungsmodelle insbesondere auch in Domänen der Sonder-, Heil-, Inklusions-, Rehabilitationsund Sozialpädagogik Ende der 1990er Jahre erweiterte die Themenvielfalt des Faches und führte zu vielschichtigen Ausdifferenzierungen, veränderten Forschungszugängen und neuen Schwerpunktsetzungen. Der anlässlich des 40-jährigen Bestehens der Sektion Sonderpädagogik in der Deutschen Gesellschaft für Erziehungswissenschaft erschienene Band über „Sonderpädagogik - Rückblicke, Bestandsaufnahmen, Perspektiven" (Schnoor \& Rohrmann, 2004) ebenso wie die Publikation zu ihrem 50-jährigen Bestehen über „Bildungs- und Erziehungsorganisationen im Spannungsfeld von Inklusion und Ökonomisierung" (Sturm, Köpfer \& Wagener, 2016) dokumentieren diese tiefgreifenden Veränderungsprozesse.

Dass die Heilpädagogischen Werkblätter auch unter dem neuen Titel VHN sich diesen Entwicklungen nicht verschlossen haben und überdies das Management 2004 in die Verantwortung des Ernst Reinhardt Verlages übergeben wurde, sollte im Rückblick als ausgesprochen zukunftsweisend wertgeschätzt werden. Mit dem neuen Verlagsort München war zugleich auch eine Erweiterung des Herausgeber/innenkreises verbunden. Ab 2004 war Haeberlin zwar verantwortlich als Gesamtherausgeber, freilich im Team mit Prof. Dr. Gérard Bless (Universität Fribourg) und Prof. Dr. Ulrich Heimlich (Ludwig-Maximilians-Universität München).

Die drei Herausgeber sprachen von einer „neue[n] Phase in der Erfolgsgeschichte der traditionsreichen Vierteljahresschrift für Heilpädagogik und ihre Nachbargebiete" (Haeberlin, Bless \& Heimlich, 2004, S. 253). Der Wechsel war verbunden mit einer organisatorischstrukturellen Präzisierung der unterschiedlichen Publikationsformate in doppelt blind peerreviewte Fachbeiträge, provokative Essays, Trends, Fachdialoge, Forschungsberichte, Buchbesprechungen u. a.m. Frau Christina Amrein hatte von 2004 bis 2010 die alleinige Redaktionsleitung inne; da sie schon vorher zusammen mit Heinz Hermann Baumgarten als VHNRedaktorin gearbeitet hatte, war sie für ihre Funktion beim Wechsel zum Reinhardt Verlag optimal vorbereitet.

Frau Hildegard Wehler sorgt als Verlagsleiterin und Geschäftsführerin des Ernst Reinhardt Verlages seit 2004 mit den Herausgeber/innen und Gutachter/innen dafür, dass sowohl die Kontinuität des Qualitätsniveaus gewährleistet bleibt als auch innovative Ideen in der $V H N$ ihren Ort finden und umgesetzt werden können.

2007 fand ein Wechsel im Herausgeberteam statt. Von 2007 bis 2016 zählten Prof. Dr. Iris Beck (Universität Hamburg) und Prof. Dr. Winfried Kronig (Universität Fribourg) nach dem Ausscheiden von Prof. Dr. Heimlich und Prof. Dr. Bless zum neuen Herausgeberteam. Herr Martin Baumgartner übernahm 2010 die Nachfolge von Frau Amrein und wurde neuer Redaktionsleiter.

Urs Haeberlin schied 2016 - nach insgesamt 33 Jahren (!) - als Geschäftsführender Herausgeber aus. Sein Anspruch, die VHN möglichst 
ideologiekritisch, jedoch - wohlverstanden nicht wertfrei zu gestalten, war das epochenprägende Momentum seiner Herausgeberschaft (vgl. Haeberlin, 2016, S. 287). Diese Programmatik zeichnet die VHN bis heute aus und soll auch den zukünftigen Fachdialog bestimmen.

Der Generationenwechsel Ende 2016 und der Beginn eines neuen Herausgeberteams war nicht frei von „Startschwierigkeiten“ (Hartmann, Herz \& Kuhl, 2017, S. 93), die allerdings nicht im Zusammenhang mit der neuen Verantwortungsübernahme standen. Bevor Prof. Dr. Erich Hartmann (Universität Fribourg) die Verantwortung als Geschäftsführender Herausgeber der VHN und Frau Prof. Dr. Birgit Herz (Leibniz Universität Hannover) zusammen mit Herrn Prof. Dr. Jan Kuhl (Technische Universität Dortmund) als Mitherausgeber/innen 2017 ihre Arbeit aufnehmen konnten, wurde nämlich die deutsche Sonderpädagogik tsunamigleich mit einem besonderen Fachkonflikt konfrontiert. Auf der Oberfläche schien es um Methoden wissenschaftlichen Arbeitens zu gehen, latent handelte es sich allerdings um Kernfragen des Faches in Bezug auf Definitionshoheit und letztendlich, damit verbunden, um Macht.

Wie in den 1980er, 1990er und den Jahren seit ,PISA' (2000) steht die sonderpädagogische Disziplin immer wieder auf dem Prüfstand derzeit besonders im Hinblick auf Ressourcen, Praxisentwicklungen sowie Theorie- und Forschungsbezüge. So konnte aktuell für die fachwissenschaftlichen Debatten und Diskurslinien mit Unterstützung von Frau Wehler bspw. der von Michael Grosche, Claudia Gottwald und Hendrik Trescher 2020 herausgegebene Band „Diskurs in der Sonderpädagogik“ im Ernst Reinhardt Verlag publiziert werden, der auf Initiative und Beteiligung des neuen Mitherausgebers Prof. Dr. Kuhl zunächst als Themenstrang in der $V H N$ vorbereitet worden war.

Zudem konnte 2018 mit der Etablierung der online-Ergänzung $V H N^{\text {plus }}$ ein neuer Impuls auf Initiative des Verlages hin - realisiert wer- den, der das Bedürfnis nach Digitalisierung im Leser/innenkreis aufgriff und zugleich ein neues Format für wissenschaftliche Fachbeiträge schuf. Um auch weiterhin die bewährte Kontinuität der Qualitätsstandards für wissenschaftliche Fachbeiträge zu gewährleisten, wurde der Kreis des wissenschaftlichen Beirats erweitert.

Die rasanten Veränderungen im Publikationsund Verlagswesen machen sich auch bei der $V H N$ und dem Ernst Reinhardt Verlag bemerkbar. Vom allgemeinen Trend des Rückgangs der Abonnent/innenzahlen bleibt die VHN nicht verschont; glücklicherweise steht dem ein wachsendes Interesse der Universitäten gegenüber, diese wichtige Fachzeitschrift in Mehrplatzlizenz zu abonnieren. Auch den Entwicklungen in der wissenschaftlichen Publikationskultur in Richtung Open Access haben sich der Verlag und das Herausgeberteam als Herausforderung gestellt: Seit 2017 bietet die VHN die Möglichkeit, Beiträge in der $V H N$ gegen Zahlung einer Publikationsgebühr open access zu publizieren; dies wird von den Autor/innen zunehmend in Anspruch genommen. Eine wichtige Rolle für die $V H N$ spielt auch die enge personelle und historisch begründete Anbindung an die Universität Fribourg und an die VAF (Vereinigung der Absolventinnen und Absolventen des Heilpädagogischen Instituts der Universität Freiburg), in der die Mitgliedschaft mit einem Abonnement der $V H N$ verknüpft ist.

Verlag und Herausgeberteam sehen optimistisch in die nächste Dekade, das bewährte Niveau des akademischen Dialoges in der Vierteljahresschrift für Heilpädagogik und ihre Nachbargebiete auch weiterhin fortzuführen. Die damit verbundene fachliche Begeisterung für die Sicherstellung der wissenschaftlichen Qualität wird auch in Zukunft auf die konstruktive Zusammenarbeit mit Kolleginnen und Kollegen angewiesen sein, die sich als Mitglieder des wissenschaftlichen Beirates engagieren. Ihnen allen sei hier ausdrücklich für ihre langjährige Verbundenheit gedankt. 


\section{Literatur}

Grosche, M., Gottwald, C. \& Trescher, H. (Hrsg.) (2020). Diskurs in der Sonderpädagogik. Widerstreitende Positionen. München: Ernst Reinhardt Verlag.

Haeberlin, U. (2016). Ein subjektiv gefärbter Rückblick des scheidenden VHN-Herausgebers. Vierteljahresschrift für Heilpädagogik und ihre Nachbargebiete, 85 (4), 277-289. https://doi.org/10. 2378/vhn2016.art37d

Haeberlin, U., Bless, G. \& Heimlich, U. (2004). Editorial der Herausgeber. Vierteljahresschrift für Heilpädagogik und ihre Nachbargebiete, 73(3), 253-254.

Hartmann, E., Herz, B. \& Kuhl, J. (Hrsg.) (2017). Editorial. Vierteljahresschrift für Heilpädagogik und ihre Nachbargebiete, 86(2), 93-95.

Herz, B. (2017). Zur historischen Proximetrie einer Wissenschaftsdisziplin. Sonderpädagogik und die Dialektik von Inklusion und Exklusion. Bad Heilbrunn: Klinkhardt.

Schnoor, H. \& Rohrmann, E. (Hrsg.) (2004). Sonderpädagogik: Rückblicke, Bestandsaufnahmen, Perspektiven. Bad Heilbrunn: Klinkhardt.

Sturm, T., Köpfer, A. \& Wagener, B. (Hrsg.) (2016). Bildungs- und Erziehungsorganisationen im Spannungsfeld von Inklusion und Ökonomisierung. Bad Heilbrunn: Klinkhardt.

\section{Anschrift der Autorin}

\section{Prof. Dr. Birgit Herz}

Leibniz Universität Hannover

Institut für Sonderpädagogik

Pädagogik bei Verhaltensstörungen

Schloßwender Straße 1

D-30159 Hannover

E-Mail: birgit.herz@ifs.uni-hannover.de 